\title{
Solar activity studies: From a magnetohydrodynamics description to a plasma perspective
}

\author{
WANG JingXiu \\ Key Laboratory of Solar Activity, National Astronomical Observatories, Chinese Academy of Sciences, Beijing 100012, China
}

Received November 15, 2011; accepted February 20, 2012

\begin{abstract}
Solar activity, in which there is an explosive release of magnetic energy in the solar atmosphere, is one of the most spectacular manifestations of space plasma activity. Non-potential, free magnetic energy is accumulated by the interaction between plasma and magnetic fields in the photosphere and sub-photospheric layer. In the photosphere and lower solar atmosphere, plasma is only partially ionized and there is three-species (or three-fluid) plasma. Cowling conductivity should therefore be considered in explaining solar observations. On the other hand, the explosive release of magnetic energy is believed to be caused by magnetic reconnection in the corona, where plasma is fully ionized and essentially collisionless. However, for collisionless plasma, we seem to have no precise quantitative description or formulation of its conductivity. To physically understand magnetic reconnection in collisionless plasma is an important task in making further progress in solar activity studies. This article discusses the plasma perspective of solar activity studies. The discussion begins with solar observations, and then, an analysis of the plasma problems that we face and need to better understand.
\end{abstract}

solar activity, magnetohydrodynamics, plasma physics, magnetic reconnection

Citation: Wang J X. Solar activity studies: From a magnetohydrodynamics description to a plasma perspective. Chin Sci Bull, 2012, 57: 1362-1368, doi: $10.1007 / \mathrm{s} 11434-012-5089-\mathrm{z}$

The Sun is a laboratory for plasma physics. All known plasma processes occur within the Sun, in either the solar interior or atmosphere. On the other hand, fundamental problems in solar physics cannot be finally resolved without progress in plasma physics [1-3]. Two examples of problems in solar physics are coronal heating and solar wind acceleration: it remains unclear why the Sun's corona is heated to millions of degrees and how the solar coronal plasma is accelerated to a speed of hundreds of kilometers per second. The physics involved in coronal heating and solar wind acceleration is universal in general astrophysics and space science. The solar corona consists of extremely hot and tenuous plasma with a penetration of complex solar magnetic fields. The same hot, tenuous, magnetized plasma is present throughout the universe. Waves, turbulence, microinstabilities, and the interaction of charged particles,

email: wangjx@nao.cas.cn magnetic fields and plasma waves play essential roles in the corona and solar wind environment [4]. This article does not deal with the above plasma problems but discusses plasma perspectives in solar activity studies; i.e., it asks: should we consider plasma kinetic processes in solar activity studies?

Solar activity is an explosive release of magnetic energy in the solar atmosphere in the forms of solar flares, filament eruptions and coronal mass ejections [5]. There are two essential questions regarding the origin of solar activity. First, how does the magnetic field in the solar atmosphere become strongly stressed or non-potential; i.e., what is the mechanism by which magnetic energy accumulates in the solar atmosphere? Second, how is the accumulated magnetic energy in the solar atmosphere explosively released?

Figure 1 illustrates a strong solar flare in the active region AR5395 which took place on March 10, 1989. This flare and the resulting disastrous space weather resulted in a power outage in Quebec, Canada for 9 hours. Superposed 


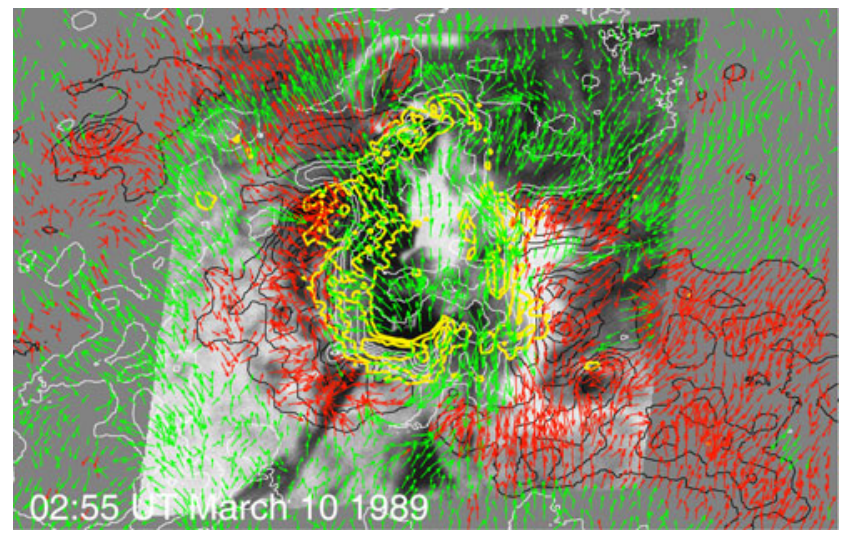

Figure 1 The strong flare (bright ribbons and dark filament eruptions) on March 10, 1989. Superposed on the flare image are vector magnetic fields with contours for the line-of-sight field component and arrows for transverse field components: white (black) contours for positive (negative) polarity; green (red) arrows from (to) positive (negative) polarity. The yellow contours indicate the free magnetic energy density in the photosphere. The vector magnetograms were obtained as Huairou Solar Observing Station of National Astronomical Observatories, Chinese Academy of Sciences.

on the flare image are vector magnetograms with contours for the line-of-sight field component and arrows for transverse field components. The flare appeared within the western periphery of a huge positive sunspot and a dark filament eruption appeared within the sunspot's eastern periphery. It is noted that the flare ribbons and plasma eruptions are closely related to the high magnetic free-energy density (see the thick yellow contours) in the photosphere where the negative magnetic flux intruded and squeezed into the positive flux of the main positive sunspot, forming severely curved magnetic interfaces. The magnetic field in the vicinity of the flare ribbons was strongly sheared and observed to be parallel to the magnetic neutral line; i.e., the polarity reversal line. The complex magnetic configuration associated with this major flare is common to other flare-prolific solar active regions [6].

The magnetic configuration and evolution associated with major flares can be described globally in the framework of magnetohydrodynamics (MHD). Even so, to completely answer the questions that arise from observations of solar active phenomena, we need a thorough understanding of the plasma problems involved.

The next section briefly describes the MHD presentation of magnetic energy accumulation and explosive release. Section 2 is devoted to an argument about what plasma perspectives of solar activity need to be considered. The final section discusses important future research efforts.

\section{MHD description of magnetic energy storage and release}

\subsection{Common environment of magnetic explosions}

From a theoretical point of view, a magnetic energy buildup and explosive release are the natural course of magnetic field evolution in many objects of astrophysics and space science, such as the Earth's magnetosphere, solar and stellar atmospheres, and accretion disks. The magnetic explosions in these different environments share common ingredients: a dense driver and a dilute magnetic corona [7].

For the solar atmosphere, the dense driver is the photosphere and the layers immediately below and above, which are referred to as the lower solar atmosphere in this paper. The driver is dynamically dominated by plasma flows and convection. The plasma property, $\beta$, which is the ratio of gas pressure to magnetic pressure, is close to 1 in the lower atmosphere. The magnetic field and plasma are in vigorous interaction, creating magnetic complexity and excess magnetic energy. However, the dilute inner corona is magnetically dominant with plasma $\beta<<1$, and the magnetic field in the corona is basically force-free. The driver and the corona are coupled via the magnetic field that stems from the interior of the Sun and penetrates through the photosphere to the corona.

In the case of magnetosphere activity, magnetic energy accumulates through the interaction of the solar wind and magnetosphere in the magnetopause, the driver, where $\beta$ is close to 1 . The explosive release of energy takes place in the domains of the ionosphere-magnetosphere-magnetotail [8], which is magnetically dominant. The magnetospheres of a solar active region and the Earth are compared in Figure 2, which is composed from diagrams of unified flare models and geomagnetic activity. It is of interest to note some overall similarity between an active region magnetosphere and the Earth's magnetosphere.

\subsection{MHD description}

We assume that the solar atmosphere is a semi-infinite three-dimensional volume with the photosphere as the lower boundary layer. The photosphere is thus the open surface through which Poynting flux enters the atmosphere. Therefore, the change in electromagnetic energy in a volume $V$ is described as

$$
\frac{\mathrm{d} W}{\mathrm{~d} t}=-\oiiint_{v} \vec{E} \cdot \vec{J} \mathrm{~d} v-\oiint_{s} \frac{1}{\mu}(\vec{E} \times \vec{B}) \cdot \mathrm{d} \vec{s}
$$

where $\mu$ is the magnetic permeability of free space, which is $1.257 \times 10^{-6} \mathrm{H} \mathrm{m}^{-1}$. The first term on the right represents the rate of change due to the work of the electric field on the electric currents within the volume, while the second represents the rate of change due to the inflow of Poynting energy flux. We further express the electromagnetic energy inflow through the photosphere by observable quantities as

$$
\frac{\mathrm{d} w_{\mathrm{inf}}}{\mathrm{d} t}=\oiint_{s}\left\{\left[B_{\perp}^{2} V_{z}-\left(\vec{V}_{\perp} \cdot \vec{B}_{\perp}\right) B_{z}\right]+\eta \vec{B} \cdot\left(\nabla B_{z}-\frac{\partial B_{\perp}}{\partial z}\right)\right\} \cdot \mathrm{d} s
$$



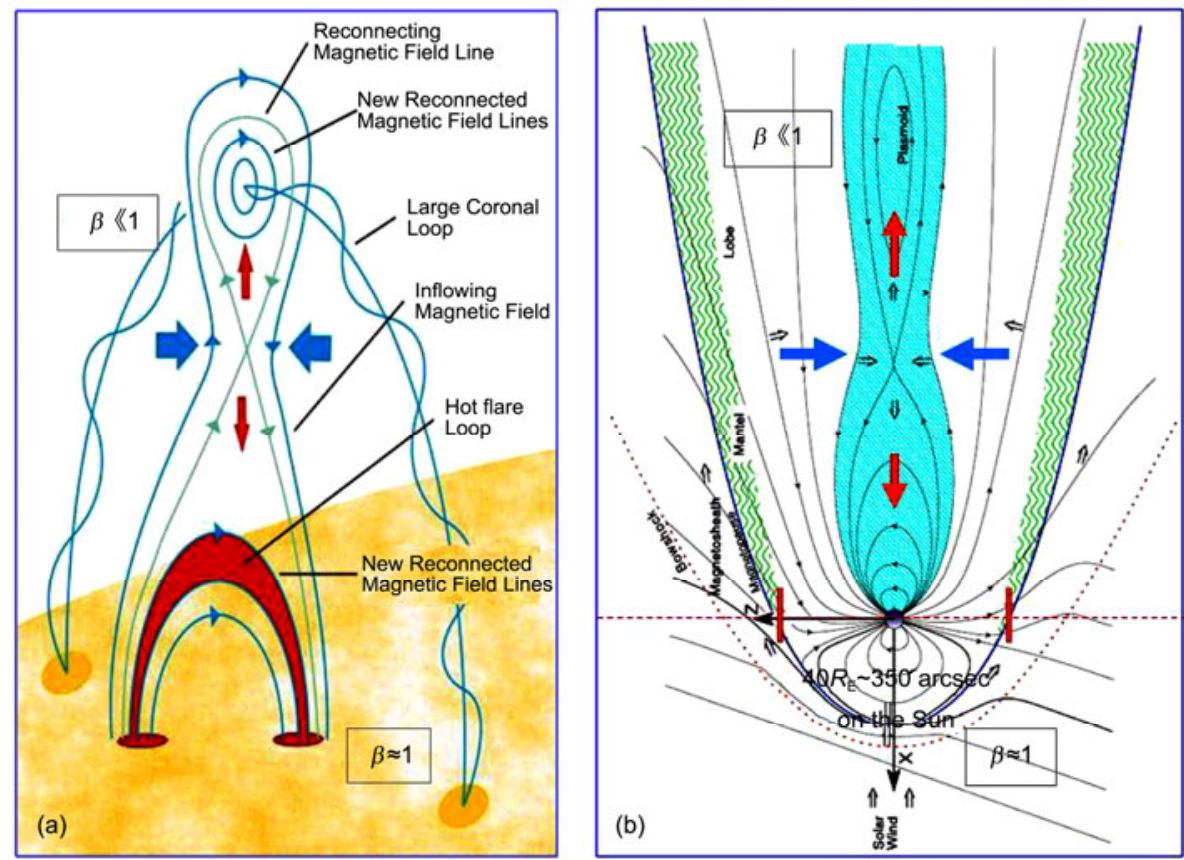

Figure 2 (a) Magnetic configuration of an active region magnetosphere and a diagram of the standard flare model; (b) magnetic configuration of the Earth's magnetosphere and a diagram of geomagnetic activity. The spatial scale has been adjusted to be roughly the same for both magnetospheres. The figure was made by combining the illustrations of the unified flare model (Kazunari Shibata) and magnetosphere model (Antonius Otto).

where $\eta$ is the magnetic diffusivity, and ' $\perp$ ' and ' $z$ ' indicate the horizontal and vertical directions relative to the local solar surface. In principle, all quantities in the formula can be observationally determined. The physics included in eq. (2) is very clear. The energy input through the open surface of the system consists of three terms: (1) flux emergence, $B_{\perp}^{2} V_{z}$, (2) shearing of the magnetic field by plasma flow, $\left(\vec{V}_{\perp} \cdot \vec{B}_{\perp}\right) B_{z}$ and (3) magnetic diffusion on the solar surface, $\eta \vec{B} \cdot\left(\nabla B_{z}-\frac{\partial B_{\perp}}{\partial z}\right)$. The formula is useful in interpreting the energy accumulation in solar active regions. Diffusion in the photosphere can transport magnetic energy into the upper atmosphere when the magnetic topology is favorable, for example the so-called 'bald patches' where the magnetic lines of force are concave upward.

It is well known that the release of magnetic energy depends on the topology complexity of the magnetic field. In terms of magnetic helicity, we can derive the complexity change in the magnetic system as [9]

$$
\frac{\mathrm{d} H_{m}}{\mathrm{~d} t}=\oiint_{s}\left[(\vec{A} \cdot \vec{V}) B_{z}-\frac{1}{\sigma}\left(A_{x} J_{y}-A_{y} J_{x}\right)\right] \mathrm{d} s-2 \eta H_{c},
$$

where $\sigma$ is the electrical conductivity, $\vec{A}$ is the vector potential of $\vec{B}$, and $H_{c}$ is the volume integral of current helicity, which is defined by $h_{c}=\frac{1}{\mu} \vec{B} \times \vec{J}$. Again, the Ohmic diffusion term has not been ignored in deducing the equa- tion. In the normal case for a rapidly growing solar active region, the last term on the right of eq. (3) can be neglected. However, we do not know if the active atmosphere has a region or regions of rapid diffusion of current helicity.

In solar activity studies, it is believed that there is explosive magnetic energy release in the corona through fast magnetic reconnection [10]. The magnetic reconnection in three dimensions is poorly known. Although the theory of two-dimensional reconnection is highly developed, many fundamental questions remain. The maximum reconnection rate in the fast Petschek's regime

$$
M_{e}^{*}=\frac{\pi}{8 \log R_{m e}}
$$

is not a physically well-understood solution but derived by specifying the ratio of the diffusion-region inflow field to that at infinity [10]. On the other hand, in MHD numerical simulations, anomalous resistivity is often arbitrarily assumed when the currents flowing in the corona reach a critical value. The physics behind this assumption is not clear [11]. However, indications of fast, impulsive reconnections are indeed observed in the higher solar atmosphere. Moreover, explosive magnetic reconnection has been studied with inspiring results in laboratories [12].

\subsection{Questions}

The Ohmic diffusion terms have not been dropped from deduced eqs. (2) and (3) as in many MHD studies. The rea- 
son for this is that there is partially ionized plasma from the photosphere to the region of temperature minimum. The atmosphere there is basically neutral with an ionization degree of only $10^{-3}-10^{-4}$. The resistivity is much greater than for fully ionized plasma. Only if the plasma effects are taken into adequate account can the MHD approaches be adopted in the lower atmosphere.

When considering the explosive release of magnetic energy in the corona, we are dealing with collisionless plasma. Without some basic understanding of the turbulence and instabilities in the collisionless plasma, the magnetic explosion in the corona cannot be satisfactorily understood.

\section{Plasma perspective}

\subsection{Partially ionized plasma in the lower atmosphere}

The lower solar atmosphere (i.e. the photosphere and layers immediately above and below) has a very low ionization degree. Figure 3 shows the ionization degree of an updated model of the solar atmosphere [13]. In the minimum-temperature region, the ionization degree is only $10^{-4}$. The conductivity of fully ionized plasma cannot be used in this case. To deduce the electric conductivity in the partially ionized plasma, we follow Cowling [1] and adopt threespecies fluid consisting of neutral atoms, electron, and ions. For a given position, when a coordinate system whose $z$ axis is parallel to the magnetic field is chosen, from the equation of motion of the combined electrons and ions, we obtain general Ohm's law in the form

$$
\vec{J}=\vec{\sigma} \cdot \vec{E}_{0}
$$

and

$$
\vec{\sigma}=\left(\begin{array}{ccc}
\sigma_{\perp} & -\sigma_{\mathrm{T}} & 0 \\
\sigma_{\mathrm{T}} & \sigma_{\perp} & 0 \\
0 & 0 & \sigma_{\|}
\end{array}\right),
$$

where $\sigma_{\|}$and $\sigma_{\perp}$ are parallel and perpendicular conductivities that refer, respectively, to the currents parallel and perpendicular to the direction of the magnetic field, and $\sigma_{\mathrm{T}}$ is the transverse or Hall conductivity that refers to the Hall current perpendicular to both the magnetic and electric fields [14]. All the conductivities are associated with the parameters of collision among the neutral atoms, electrons, and ions. In the following expressions, subscripts $n, e$, and $i$ indicate the quantities of atoms, electrons, and ions, respectively.

$$
\begin{gathered}
p=\frac{\tau_{e i}}{(1+\alpha) \tau_{e n}}, \\
q=\frac{\alpha f^{2} \varpi_{e}^{2} \tau_{e i} \tau_{e n}}{(1+\alpha)}, \\
r=\varpi_{e} \tau_{e i}\left(1-\frac{2 \alpha f}{1+\alpha}\right),
\end{gathered}
$$

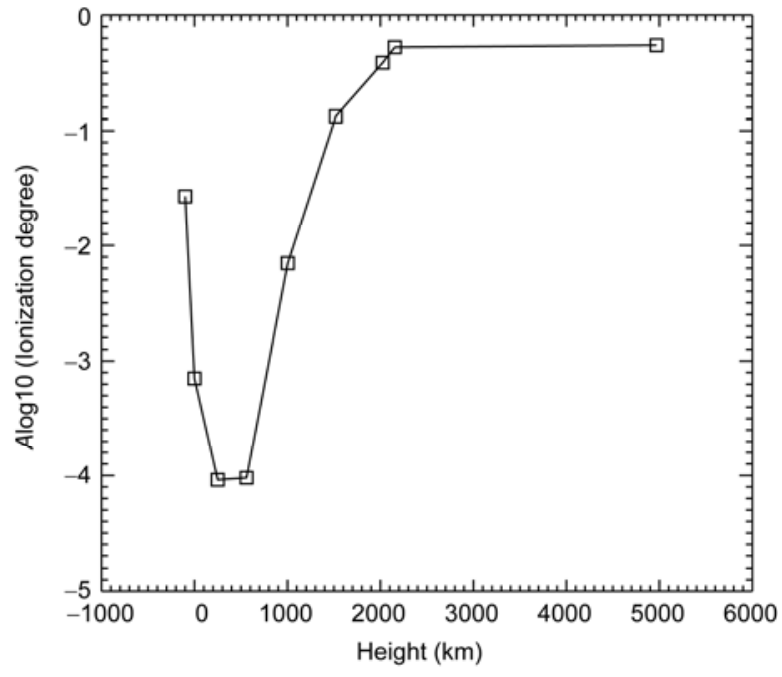

Figure 3 Ionization degree of a model of the solar atmosphere.

where $\varpi_{e}=\frac{e B}{m_{e}}, f=\frac{n_{e}}{n_{n}+n_{e}}, \alpha=\frac{2 m_{e} \tau_{i n}}{m_{i} \tau_{e n}}$, and $\tau$ is the collision interval for any two of the three species. The conductivities are then

$$
\begin{aligned}
\sigma_{\|} & =\sigma_{0}(1+p)^{-1}, \\
\sigma_{\perp} & =\sigma_{0}(1+p+q)^{-1}\left[1+r^{2}(1+p+q)^{-2}\right]^{-1}, \\
\sigma_{\mathrm{T}} & =\sigma_{\perp} r(1+p+q)^{-1}, \\
\sigma_{0} & =\frac{n_{e} e^{2} \tau_{e i}}{m_{e}}
\end{aligned}
$$

which can be readily estimated from the plasma parameters. Here, $\sigma_{0}$ is the scalar conductivity for fully ionized plasma. The conductivity in the partially ionized plasma follows the relation $\sigma_{0}>\sigma_{\|}>\sigma_{\perp} \sim \sigma_{\mathrm{T}}$. We deduce the rate of current dissipation (i.e., the rate of Ohmic diffusion) as

$$
\vec{J} \cdot \vec{E}=\vec{J} \cdot(\vec{\rho} \cdot \vec{J})=\frac{J_{\|}^{2}}{\sigma_{\|}}+\frac{J_{\perp}^{2}}{\sigma_{\perp}^{*}},
$$

where $\vec{\rho}$ is the tensor of resistivity (i.e., the inversion of the conductivity tensor), $\sigma_{\perp}^{*}$ is the effective perpendicular conductivity, often called Cowling conductivity in the literature, and $\sigma_{\perp}^{*}=\sigma_{\perp}+\frac{\sigma_{\mathrm{T}}^{2}}{\sigma_{\perp}}$. Before considering the application of the above results to the lower atmosphere, we present two discussions of interest.

(i) When the magnetic field is weak, the conductivity tensor is isotropic and $\vec{\sigma}=\sigma_{0}^{*} \vec{I}$, where $\sigma_{0}^{*}$ is a scalar conductivity and $\sigma_{0}^{*} \approx \sigma_{\|} \approx \sigma_{\perp}$. In this case, there is no Hall conductivity; i.e., $\sigma_{\mathrm{T}}=0$. The only revision of the conductivity comes from $\sigma_{0}^{*}=\sigma_{0}(1+p)^{-1}$. We have ap- 
proximately for weakly ionized plasma

$$
\sigma_{0}^{*} \propto Z T^{-2}
$$

where $Z$ is the ionization degree of the atmosphere. A lower ionization degree means more collisions between charged particles with neutral atoms, which would seriously reduce conductivity.

(ii) When the magnetic field is non-negligible, the parallel conductivity is not affected by the presence of the magnetic field, but there is a strong modification of perpendicular and Cowling conductivity. When the contribution of $q$ is relatively large, we have roughly

$$
\frac{\sigma_{\perp}}{\sigma_{0}} \propto B^{-2} .
$$

The perpendicular and Cowling conductivity would be much less than the fully ionized scalar conductivity.

Wang [14] estimated conductivity in the partially ionized solar atmosphere based on a few earlier solar atmospheric models. The preliminary conclusions are as follows. (1) The Ohmic diffusion in the lower atmosphere cannot be ignored, and the conductivity in the zone of temperature minimum might be small enough to account for the rate of flux disappearance in the observed flux cancellation [15]. (2) If there is any mechanism or mechanisms to generate perpendicular electric currents, the Ohmic diffusion would be fast enough to heat the atmosphere or even trigger activity in virtue of very low Cowling conductivity. New estimations by adopting the updated atmosphere models and more carefully deducing conductivity in partially ionized plasma are needed. A detailed discussion on partially ionized plasma can be found in Parker [16].

Ji and Song [17] and Ji et al. [18,19] studied magnetic reconnection in partially ionized plasma. These authors recognized that owing to the finite resistivity near the current sheet, the "usual MHD description must fail, and we need another theory to join in, such as a kinematic description, to account for violent phenomena (e.g., Ellerman bombs and type II white light flares) that occur in the Sun's lower atmosphere". They interpreted the observed magnetic flux cancellation by the magnetic reconnection in the partially ionized plasma.

Arber et al. [20] studied the emergence of a flux tube through a partially ionized solar atmosphere. They recognized that for a magnetic flux tube, or any magnetic flux, to emerge into the solar corona from the convection zone, it must pass through the partially ionized layers of the lower atmosphere: the photosphere and the chromosphere. In such regions, collisions with neutral atoms increase the resistivity for currents flowing across magnetic field lines, as illustrated earlier by Wang [14]. These authors declared that simulations of flux emergence should include such a neutral layer to obtain the correct perpendicular current, remove the Rayleigh-Taylor instability, and obtain the correct temperature profile.

\subsection{Collisionless plasma in the corona}

The conditions of the average corona are given in Table 1 with some key plasma quantities [2]. The density is lower and the collisions become fewer in the corona so that the mean-free path for binary collision between particles becomes much greater than the characteristic scale $L$ of the system. Both the plasma parameter and the Lundquist number are very large. Therefore, the plasma in the corona is essentially collisionless. Completely collisionless plasma is found in solar wind and the Earth magnetosphere [10]. The resistivity of collisionless plasma can be ignored in the macroscopic dynamics of the global scale $L$. However, as Schindler [2] argued, the resistive processes associated with scales much smaller than $L$ might play an important role in the system. The coronal plasma cannot strictly be treated with traditional MHD. Although this has been widely understood by solar astronomers, fewer advances have been made for a complete description of collisionless plasma in the corona in solar activity studies. An accurate description of collisionless plasma is not presented in this paper owing to the restricted scope of the paper and knowledge of the author.

The so-called standard flare model suggests that the magnetic explosion in solar flares and CMEs is caused by explosive magnetic reconnection in the corona. However, the reconnection should require greatly enhanced resistivity. It is still an open question: what is the origin of the enhanced resistivity in the small-scale structure, e.g., current sheets, in collissionless plasma and what initiates this explosive magnetic reconnection [21].

From an observational point of view, Moore and Roumeliotis [22] and Wang and Shi [15] considered the coupling of the lower atmosphere and higher atmosphere by the magnetic field that would tie together the charged particles in the plasma. They argued for the important role of slow reconnection in the lower atmosphere of finite conductivity in creating the conditions for the fast reconnection in the collisionless corona. Wang and Shi proposed a two-step reconnection scenario, according to which slow reconnection in the lower atmosphere would finally result in explosive reconnection in the corona. Moore and Roumeliotis clearly stated that reconnection in the lower atmosphere would trigger explosive magnetic reconnection in the dilute corona [22]. Tsurutani et al. [8] proposed two-step reconnection models for both the solar flare and magnetosphere substorm. However, all these authors failed to provide criterion conditions for triggering the magnetic explosion in the coronal

Table 1 Plasma parameters in the corona ${ }^{\text {a) }}$

\begin{tabular}{ccccccccc}
\hline$T_{e}(\mathrm{~K})$ & $L(\mathrm{~m})$ & $B(\mathrm{~T})$ & $n\left(\mathrm{~m}^{-3}\right)$ & $\omega_{p}\left(\mathrm{~s}^{-1}\right)$ & $l_{e}(\mathrm{~L})$ & $\tau_{e i}(\mathrm{~s})$ & $\Lambda_{p}$ & $S$ \\
\hline $2 \times 10^{6}$ & $3 \times 10^{7}$ & $1 \times 10^{-2}$ & $1 \times 10^{15}$ & $2 \times 10^{9}$ & $2 \times 10^{-2}$ & $7 \times 10^{-2}$ & $1 \times 10^{8}$ & $5 \times 10^{14}$ \\
\hline a) $l_{e}$, the electron mean free path, $\Lambda_{p}$, plasma parameter, $S=\tau_{D} / \tau_{A}$,
\end{tabular}
Lundquist number.


collisionless plasma. How does the reconnection with collisional resistivity in the partially ionized plasma result in the explosive reconnection in collisionless plasma? This appears to be an essential question in the studies of space plasma activities.

It is noticed that both two types of magnetic reconnection, collisional and collisionless, have been well identified through numerail and laboratory simulations [12,23]. It has been revealed that the reconnection rate in collisionless plasma depends on the plasma $\beta$ and the thickness of the diffusion region. In a simulation of steady state Hall MHD reconnection, the "two-step reconnection" behavior has been identified and illustrated [24]. With the increase of the scaling parameter $d_{i} / L_{c}$, in which $d_{i}$ is the ion inertial length and $L_{c}$ is the width of Harris current sheet, there appears a transition from the scheme of classical resistivity-dependent reconnection to that of collisionless fast reconnection. Assume $\beta=0.5$ and adopt a flux inflow $V_{\text {in }}>0.01$, when $d_{i}<<$ $L_{c}$ the dimensionless reconnection rate is 0.015 and it is only related to the resistivity. However, when $L_{c} \leqslant 10 d_{i}$, the collisionless Hall effect becomes dominant, and the reconnection rate increases, which only depends on the thickness of current sheet and plasma beta (see Figures 1 and 2 in [24]). In a well-scaled laboratory experiment, the megagauss-scale magnetic field, produced by interaction of a high-intensity laser with plasma, manifests a magnetic reconnection topology [25]. The topology is similar to the general magnetic environment for solar flares. In the setup of the experiment the ionization degree is not very high and the plasma $\beta$ has large and rapid changes. It is very interesting to see the central collisionless reconnection and that with collision in the outskirts. In the collisionless reconnection ions become decoupled from electrons on scale of ion inertial length.

An accurate understanding of magnetic reconnection in collisionless plasma requires a reliable treatment of the resistivity in terms of the generalized Ohmic law within the framework of the Vlasov regime. Early in the 1970s general Ohm's law was given [26]. Here we adopt the detailed version in Schindler [2] with a slight modification, as

$\vec{E}+\vec{v} \times \vec{B}=-\frac{1}{e n_{e}} \nabla \cdot \vec{P}_{e}+\frac{\vec{J} \times \vec{B}}{e n_{e}}-\frac{m_{e}}{e}\left(\frac{\partial \vec{v}_{e}}{\partial t}+\vec{v}_{e} \cdot \nabla \vec{v}_{e}\right)+\frac{M_{e}^{\text {(add) }}}{e n_{e}}$,

where $\vec{J}=e n_{i} \vec{v}_{i}-e n_{e} \vec{v}_{e}$ and $\vec{v}=\frac{n_{i} m_{i} \vec{v}_{i}+n_{e} m_{e} \vec{v}_{e}}{n_{i} m_{i}+n_{e} m_{e}}$ are, respectively, the current density and plasma bulk flow velocity, and $M_{e}^{\text {(add) }}$ is an additional term leading to the momentum transfer between electrons and ions. The right side of the equation represents the non-ideal terms: from left to right are the pressure term, the generalized Hall term, the electron inertia term, and the resistive term due to electron-ion momentum transfer. In deduction this equation, the quasi-neutrality and $m_{e}<<m_{i}$ are assumed. Eq. (11) pro- vides a framework to deal with resistivity. When the binary collision is dominant, $M_{e}^{(\text {add })}=M_{e}^{(b c)}$, then

$$
\frac{\vec{M}_{e}^{(b c)}}{e n_{e}}=\eta \vec{J}
$$

for the collisional plasma. In the collisionless plasma, microturbulence is the main cause of resistance. From a macroscopic point of view, the integrative effect of the microturbulence may be the cause of turbulent or collective transport, thusly $M_{e}^{(\text {add) }}=M_{e}^{\text {(turb) }}$, and we have

$$
\frac{\vec{M}_{e}^{\text {(turb) }}}{e n_{e}}=\eta_{\text {turb }} \vec{J}
$$

provided that $\vec{M}_{e}^{\text {(turb) }}$ and $\vec{J}_{\text {turn }}$ are parallel. We then have the turbulent or collective resistivity $\eta_{\text {turb }}$. However, the accurate description of $\vec{M}_{e}^{\text {(turb) }}$ is generally not known. Either the collision resistivity or the turbulent resistivity is associated with the fluctuation of the electric field that induced momentum transfer between electrons and ions. The most difficult task is getting reliable knowledge of the fluctuations in the collisionless plasma.

The observational diagnosis and theoretical study of plasma instabilities, in particular, the instabilities that lead to microturbulence in the collisionless plasma, is of particular importance in future solar studies. The importance lies not only in the studies of magnetic explosions in the corona but also in the fundamental research on coronal heating. So far we have examined the case of partially ionized plasma but have no enough knowledge about the resistivity associated with the magnetic explosion in collisionless plasma. There is little consensus on the processes that result in the non-idealness, i.e., break the fronzen-in condition in collisionless reconnection. A question is whether there appears enhanced resistivity or there are processes to free plasma from slavery by the magnetic field [27].

\section{Future efforts}

Solar astronomers now have great opportunities and challenges in solar activity studies. With the Solar Optical Telescope aboard Hinode [28], we can observed the solar vector magnetic field with unprecedented spatial resolution and adequate polarization sensitivity. Moreover, full-disk Doppler images and vector magnetograms have been obtained with the Helioseismic and Magnetic Imager onboard the Solar Dynamics Observatory with high cadence, consistent sensitivity and continuous temporal coverage [29]. This is the first time that solar astronomers can observe the Sun's magnetism and magnetohydrodynamic processes in great detail. Furthermore, the Atmospheric Imaging Assembly aboard the Solar Dynamics Observatory provides multiple simultaneous high-resolution full-disk images of the corona 
and transition region up to $0.5 R$ above the solar limb with 1.5 -arcsec spatial resolution and 12-second temporal resolution [30]. We now have good facilities to diagnose coronal plasma. In the future, imaging spectrograph from space will be a more powerful tool in the diagnosis of plasma properties.

From an observational point of view, a few efforts will be important. First, matching the vector magnetic field observations with atmospheric structures in multiple-temperature domains will provide clues on how the magnetic field ties the plasma together and couples the atmosphere from the photosphere and chromosphere to the corona. Secondly, identifying the magnetic connectivity in active region scale and also in large or even global scale will help obtain an accurate understanding of the accumulation and release of magnetic energy through the evolution and interaction of multi-flux systems, and help obtain a true picture of magnetic reconnection. Thirdly, diagnosing the plasma properties and instabilities with multi-wavelength spectral and imaging observations will lead to better knowledge of the turbulent resistivity and the triggering of the release of explosive magnetic energy during solar activity.

From a theoretical point of view, future progress in solar activity studies cannot be made without significant advances in plasma physics. As we discussed previously, for either partially ionized plasma or hot and dilute collisionless plasma, traditional MHD treatment is not sufficient. We solar astronomers should work with plasma physicists to identify the key plasma problems behind detailed observations and learn the newest results of their numerical simulations and laboratory experiments.

We are fully aware that in the foreseeable future it will be difficult to completely resolve the problems, raised in this paper. MHD theory with input of kinetic plasma theory would provide a basic theoretical framework for understanding the physics of solar and astrophysical magnetic activity. MHD theory and kinetic plasma theory are seen to serve as the two wings of current solar and space research.

The author appreciates the valuable comments and suggestions made by the referee, and thanks Yan Song and Chijie Xiao for helpful discussions. This work was supported by the National Natural Science Foundation of China (11003024, 40974112, 10973019, 10921303, 11025315) and the National Basic Research Program of China (G2011CB811403).

1 Cowling T G. Magnetohydrodynamics. Bristol: Adam Hilger, Ltd., 1976

2 Schindler K. Physics of Space Plasma Activity. New York: Cambridge University Press, 2007

3 Huang G. Plasma Kinematics and Its Application to Solar Physics (in Chinese). Beijng: Science Press, 2009

4 Wu D J, Fang C. Two-fluid motion of plasma in Alfvén waves and the heating of solar coronal loops. Astrophys J, 1999, 511: 958-964

5 Fang C, Ding M D, Chen P F. Physics of Solar Active Regions (in Chinese). Nanjing: Nanjing University Press, 2008

6 Wang J. Vector magnetic fields and magnetic activity on the Sun.
Fund Cosmic Phys, 1999, 20: 251-382

7 Kuijpers J. Physics of Flares in Stars and Accretion Disks. In: Schmelz J T, Brown J C, eds. The Sun: A Laboratory for Astrophysics. Dordrecht: Kluwer Academic Publishers, 1992

8 Tsurutani B T, Shibata K, Akasofu S I, et al. A two-step scenario for both solar flares and magnetospheric substorms: Short duration energy storage. Earth Planets Space, 2009, 61: 555-559

9 Wang J. A note on the evolution of magnetic helicity in active regions. Solar Phys, 1996, 163: 319-325

10 Priest E, Forbes T. Magnetic Reconnection. New York: Cambridge University Press, 2000

11 Wu G P, Huang G L, Ji H S. Dependence of anomalous resistivity on bulk drift velocity of electrons in the reconnecting current sheets in solar flares. Res Astron Astrophys, 2010, 10: 1186-1194

12 Yamada M. Progress in understanding magnetic reconnection in laboratory and space astrophysical plasma. Phys Plasmas, 2007, 14: 058102

13 Averett E H, Loeser R. Models of the solar chromosphere and transition region from SUMER and HRTS observations: Formation of the extreme-ultraviolet spectrum of hydrogen, carbon, and oxygen. Astrophys J Suppl Ser, 2008, 175: 229-276

14 Wang J. Electric conductivity of lower solar atmosphere. ASP Conf Ser, 1993, 46: 465-468

15 Wang J, Shi Z. The flare-associated magnetic changes in an active region. II. Flux emergence and cancellation. Solar Phys, 1993, 143: 119-139

16 Parker E N. The alternative paradigm for magnetospheric physics. J Geophys Res, 1996, 101, 10587-10626

17 Ji H S, Song M T. Three-dimensional solutions for fan and spine magnetic reconnection in partially ionized plasmas. Astrophys $\mathrm{J}$, 2001, 556: 1017-1026

18 Ji H S, Song M T, Li X Q. Current-sheet buildup and magnetic reconnection in weakly ionized solar lower atmosphere. Solar Phys, 2001, 198: 133-148

19 Ji H S, Song M T, Huang G L. Exact solutions for two-dimensional steady state magnetic reconnection in partially ionized plasmas. Astrophys J, 2001, 548: 1087-1092

20 Arber T D, Haynes M, Leake J E. Emergence of a flux tube through a partially ionized solar atmosphere. Astrophys J, 2007, 666: 541-546

21 Aschwanden M. Physics of the Solar Corona: An Introduction with Problem and Solutions. New York: Springer Praxis, 2009

22 Moore R L, Roumeliotis G. Triggering of eruptive flares - Destabilization of the preflare magnetic field configuration. Lec Notes Phys, 1992, 399: 69-78

23 Bhattacharjee A. Impulsive magnetic reconnection in the Earth's magnetotail and the solar corona. Ann Rev Astron Astrophys, 2004, 42: 365-384

24 Wang X, Yang H A, Jin S P. Scalings of steady state hall magnetohydrodynamics reconnection in high-beta plasma. Phys Plasma, 2006, 13: 060702

25 Zhong J, Li Y, Wang X, et al. Modelling loop-top X-ray source and reconnection outflows in solar flares with intense lasers. Nat Phys, 2010, 6: 984-987

26 Rossi B, Olbert S. Introduction to the Physics of Space. New York: McGraw-Hill, 1970

27 Treumann R A. Origin of resistivity in reconnection. Earth Planets Space, 2001, 53: 453-462

28 Tsuneta S, Ichimoto K, Katsukawa Y, et al. The solar optical telescope for the Hinode mission: An overview. Solar Phys, 2008, 249: 167-196

29 Couvidat S, Schou J, Shine R A, et al. Wavelength dependence of the helioseismic and magnetic imager (HMI) instrument onboard the solar dynamics observatory (SDO). Solar Phys, 2012, 275: 285-325

30 Lemen J R, Title A M, Akin D J, et al. The atmospheric imaging assembly (AIA) on the solar dynamics observatory (SDO). Solar Phys, 2012, 275: 17-40

Open Access This article is distributed under the terms of the Creative Commons Attribution License which permits any use, distribution, and reproduction in any medium, provided the original author(s) and source are credited. 